Article

\title{
CT-Dose Measurement of the Spinal Cord Region Using XR-QA2 Radiochromic Films and TLD 100H Dosimeters
}

\author{
Martina Pace ${ }^{1,2}$, Giuseppe Stella ${ }^{1,2, *}$, , Letizia Barone Tonghi ${ }^{2,3}$, Stefania Mazzaglia ${ }^{1,2}$ \\ and Anna Maria Gueli ${ }^{1,2}$ (D) \\ 1 PH3DRA labs, Dipartimento di Fisica e Astronomia "Ettore Majorana", Umiversità di Catania \& \\ INFN-CHNet Sez CT, via Santa Sofia 64, 95123 Catania, Italy; martina.pace@ct.infn.it (M.P.); \\ stefania.mazzaglia@ct.infn.it (S.M.); anna.gueli@ct.infn.it (A.M.G.) \\ 2 Scuola di Specializzazione in Fisica Medica, Dipartimento di Scienze Mediche Chirurgiche e Tecnologie \\ Avanzate “G. F. Ingrassia”, Scuola Facoltà di Medicina, Via Santa Sofia 78, 95123 Catania, Italy \\ 3 P.O. Garibaldi Nesima, Via Palermo, 636, 95122 Catania CT, Italy; lebatonghi@hotmail.com \\ * Correspondence: giuseppe.stella@ct.infn.it
}

Received: 17 December 2019; Accepted: 24 June 2020; Published: 3 July 2020

check for updates

\begin{abstract}
The European Directive 2013/59/Euratom focuses on the radiation protection and patient dosimetry. In particular, the dose absorbed by serial organs such as the spinal cord due to diagnostic exams plays a crucial role in the appraisal of medical exposure. In this work, a comparison between dose measurements performed with Gafchromic ${ }^{\mathrm{TM}}$ XR-QA2 and TLD 100H is presented. The dosimeters-after a calibration procedure through an X-ray tube-were placed within the thorax region of an anthropomorphic phantom corresponding to the spinal cord area exposed to a thoracic CT procedure. The mean-dose value was measured with Gafchromic ${ }^{\mathrm{TM}}$ first, and it was then compared to the dose value obtained with TLDs, resulting in a good agreement between the two dosimetric methodologies. Additionally, the results showed that-due to the usage of the automatic exposure control (EC) system - the discrepancy between the two methods is proportional to the current output of the CT system.
\end{abstract}

Keywords: 2D-dose distribution; thermoluminescence; radiodiagnostic

\section{Introduction}

The evolution and improvement of imaging techniques has contributed to increasing the number of examinations carried out during the hospital routine: clinical imaging is a fundamental tool employed by specialists in the diagnosis of various diseases. However, wide use of these techniques has led to an increase in the exposure to ionizing radiation, highlighting the dangers and the associated risks. As of today, one of the most-employed clinical imaging techniques is the Computed Tomography (CT) [1].

Over the past years, considerable effort has been put into addressing the assessment the organ doses for many different types of CT scanners (cone- and fan-beam) using different CT scan protocols and dosimetry systems such as, Gafchromic ${ }^{\text {TM }}$ and TLD crystals $[2,3]$.

Although TLD dosimeters can be used to obtain punctual information of the absorbed dose, they do not allow for accurate measurements of strong localized dose gradients-especially when multi-slice scanners are employed [4]. The TLD 100H (LiF: Mg, Cu, P) dosimeters are the most-used in clinical dosimetry and in applications where high sensitivity at low doses is needed.

The TLD-100H dosimeters have been characterized in terms of glow curve deconvolution, energy and angular dependence [5-7]. In fact, it has been found that TLD-100H dosimeters are characterized 
by a linear response inside the diagnostic energy interval and their sensitivity variation is under $3 \%$ at tube potentials ranging from $40 \mathrm{kVp}$ to $125 \mathrm{kVp}$ [5].

Radiochromic films are employed to obtain two-dimensional dose distributions with high-resolution [2,8-10]. The Gafchromic ${ }^{\mathrm{TM}} \mathrm{XR}-\mathrm{QA} 2$ is the most recent radiochromic film used to carry out measurements of CT dose distribution [3]. In general, the XR-QA model allows high-resolution slice-by-slice dose distribution measurements inside an organ volume, as well as spatial- and punctual-dose measurements, providing a potential tool to perform 2D- and 3D-CT dosimetry [2,11].

Recent studies have shown that the difference in dose response between XR-QA films and TLD arise when the film surface sensitive area is parallel to the central axis of the CT beam [2]. In addition, further studies have shown that-under certain configurations-the angular dependence of the XR-QA films can introduce significant uncertainties [2,12]. Nonetheless, it was established that a complete CT scan introduces a $2 \%$ overall uncertainty [2].

The aim of this work is to carry out a comparison between measurements performed with Gafchromic $^{\text {TM }}$ XR-QA2 and TLD 100H, placed within an inhomogeneous anthropomorphic phantom and exposed to a thoracic CT scan. The radiochromic films and the TLDs were placed within the thorax region corresponding to the spinal cord region.

\section{Materials and Methods}

\subsection{Dosimeters}

The TLD $100 \mathrm{H}$ is a thermoluminescent dosimeter suited for medical physics dosimetry applications; it is dose sensitive inside the range $1 \mathrm{pGy}-10 \mathrm{~Gy}$ and its size is $3.2 \times 3.2 \times 0.89 \mathrm{~mm}^{3}$, as reported in the Table 1 (www.thermofisher.com).

Table 1. TLD $100 \mathrm{H}$ specifications.

\begin{tabular}{cccc}
\hline $\begin{array}{c}\text { Emission Wavelength } \\
(\mathbf{n m})\end{array}$ & Relative Energy Response & Size $\left.\mathbf{( m m}^{\mathbf{3}}\right)$ & Sensitivity \\
\hline 400 & 0.98 & $3.2 \times 3.2 \times 0.89$ & 1 pGy-10 Gy \\
\hline
\end{tabular}

The emission of luminescence from TLD 100H were measured using a Risø TL/OSL-DA-10 reader. The 9635QA photomultiplier tube combined with Corning 7-59 and Schott BG-12 optical filters were used to detect luminescence emission.

The Gafchromic ${ }^{\mathrm{TM}}$ XR-QA2 film is designed as a quality-assurance (QA) tool for radiology and it is radiation sensitive within the range $20-200 \mathrm{kVp}$ and dose sensitive within the range $0.1-20 \mathrm{cGy}$ (http://www.ashland.com). It is a reflective-type film consisting of five layers: a $25-\mu \mathrm{m}$-thick radiation-sensitive layer is stacked to a $97-\mu \mathrm{m}$-thick white and reflective polyester by means of a 3 - $\mu \mathrm{m}$-thick surface layer. A $97-\mu \mathrm{m}$-protective yellow polyester is over-coated on the top of the sensitive layer by means of a $15-\mu \mathrm{m}$-thick pressure sensitive adhesive.

\subsection{Calibration Dosimeter Procedures}

Irradiation was delivered with a Samsung GC 80 X-ray tube by employing an experimental setup characterized by the following: $\Delta \mathrm{V}=140 \mathrm{kVp}, \mathrm{HVL}=5.7-\mathrm{mm} \mathrm{Al}$, source-detector distance $\mathrm{SDD}=60 \mathrm{~cm}$, a $10 \times 10 \mathrm{~cm}^{2}$ open field. The calibration of the X-ray tube was performed in terms of radiation output in air (mGy/mAs) by employing an DCT10-RS/Lemo ionization chamber connected to its electrometer (RTI Electronics, Montville, NJ, USA).

In order to produce the calibration curves for the dosimeters, for each dose point three Gafchromic $^{\mathrm{TM}}$ samples $(15 \mathrm{~mm} \times 15 \mathrm{~mm})$ and three TLDs were irradiated in air with growing dose values. 
The radiochromic films image acquisition was performed before the irradiation and $72 \mathrm{~h}$ after the irradiation [11,13], using an Epson Expression 10,000 XL flatbed scanner. The scanner that we employed has a negligible sensitivity variation in the central region that is used for the reading. This was set up using the reflective mode because of the film opaque property $[2,10,14]$, an RGB image with a resolution of $72 \mathrm{dpi}$ in 48-bit and no color corrections was applied [11,15]. The image analysis was performed in the red channel and the pixel-by-pixel background fog subtraction method was applied [11,16] using ImageJ software (available at https://imagej.net).

The TLDs signals after irradiation were acquired with a heating rate of $1 \mathrm{~K} / \mathrm{s}$ from RT to $513.15 \mathrm{~K}$ in a nitrogen atmosphere, in order to prevent sensitivity changes due to high temperature. The signal bleaching before irradiation was performed by heating the TLD in the oven at $513.15 \mathrm{~K}$ for $10 \mathrm{~min}$ and by cooling it down at room temperature $[17,18]$. During the luminescence acquisitions, the laboratory was illuminated by using Ilford DL10 lamps filtered with Ilford \#902 [19,20] in order to avoid any optical bleaching [20].

\subsection{CT Measurements}

An Alderson Rando ${ }^{\mathrm{TM}}$ woman anthropomorphic phantom was used in order to imitate the geometry of an adult woman patient. This phantom consists of 11 numbered transverse sections; each $25 \mathrm{~mm}$ thick (Figure 1a). The different holes present in each slab were filled with tissue-equivalent material (Figure 1b).
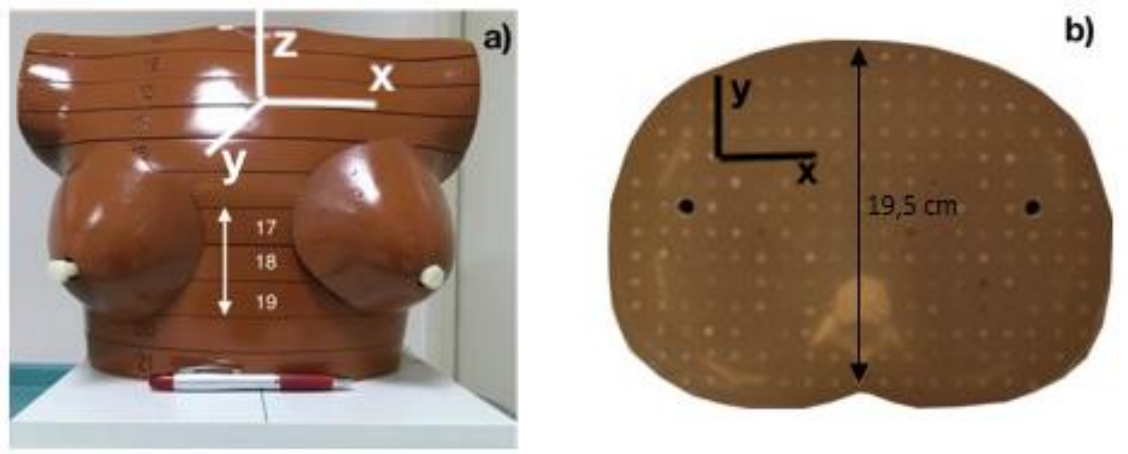

Figure 1. Alderson Rando ${ }^{\mathrm{TM}}$ woman anthropomorphic phantom: (a) front view and measurement area; (b) top view of a slab. The scan axis is along the $\mathrm{z}$ direction while $\mathrm{x}-\mathrm{y}$ is the axial plane.

XR-QA2 films samples were cut in $4 \mathrm{~cm} \times 8 \mathrm{~cm}$ pieces and applied onto the surface of the 17th, 18th and 19th phantom slabs, as in Figure 2a-c. A CT scan was then carried out. In particular, a four-slice CT scanner (GE Lightspeed) was used to scan the area between the 17th and 19th phantom slabs along the $z$-axis. The scan parameters were helical mode, AEC system, isocenter in the 18th phantom slab; length of scan equal to $153.75 \mathrm{~mm} ; 3.75 \mathrm{~mm}$ slice-thickness; $140 \mathrm{kVp}$ and pitch equal to 1. The same CT scan was repeated other 5 times, after placing the TLDs on the surface of the 17th, 18th and 19th slabs, in the position identified in Figure $2 \mathrm{a}-\mathrm{c}$, by the white points. 


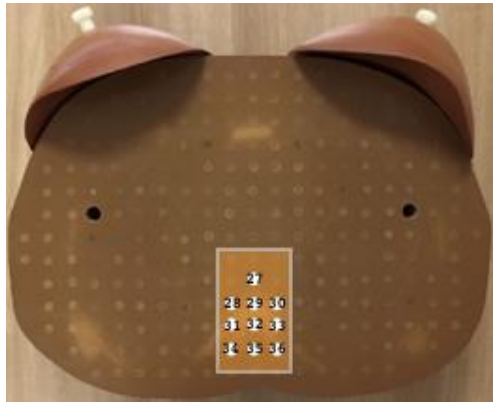

(a)

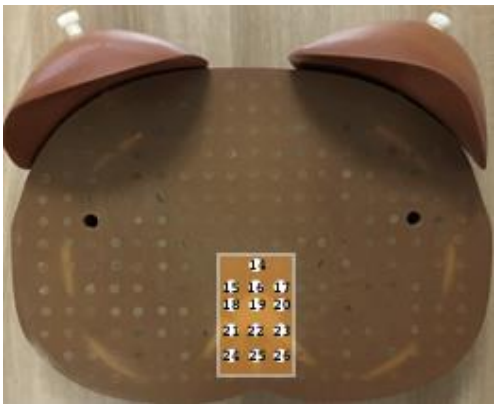

(b)

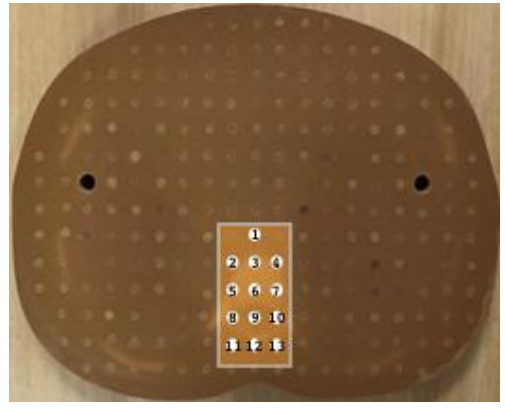

(c)

Figure 2. Position of radiochromic films (orange squares) and TLDs (white points) on the axial plane of the phantom slabs. (a) 17th slab: numbered positions from 27 to 36; (b) 18th slab: numbered positions from 14 to 26 ; (c) 19th slab: numbered positions from 1 to 13 .

\section{Results and Discussion}

\subsection{Dosimeter Dose Calibrations}

The estimation of the X-ray tube output factor and the delivered-dose values for Gafchromic ${ }^{\mathrm{TM}}$ and TLDs calibration procedures are reported in Table 2.

Table 2. Calibration step: X-ray-tube output and dose values.

\begin{tabular}{cc}
\hline Tube Output (mGy/mAs) & Dose $(\mathrm{mGy})$ \\
\hline & $9.11 \pm 0.19$ \\
& $18.23 \pm 0.38$ \\
$0.45 \pm 0.01$ & $36.46 \pm 0.77$ \\
& $57.41 \pm 1.03$ \\
& $72.89 \pm 1.37$ \\
& $91.12 \pm 1.80$ \\
\hline
\end{tabular}

Figure 3 shows the $\mathrm{PVi}_{\text {sub }}$ values as a function of the delivered-dose values shown in Table 2. The $P V_{\text {isub }}$ values (red box) represent the measured pixel values subtracted from the background and obtained from a squared ROI (side of 15 pixels).

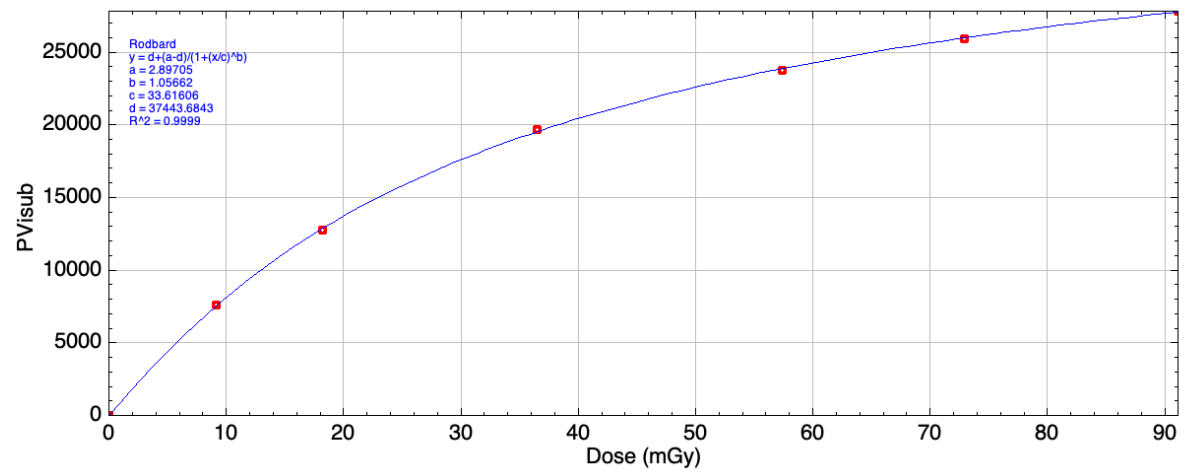

Figure 3. Calibration curve for XR-QA2 Gafchromic ${ }^{\mathrm{TM}}$.

The data were fitted (blue curve) by the equation:

$$
y=d+\frac{a-d}{1+\left(\frac{x}{c}\right)^{b}}
$$


obtaining a $R^{2}$ correlation factor equal to 0.9999 .

Figure 4 shows the trend of the TL intensity versus the dose values. The mean TL intensity, including standard deviation, is represented with black squares while for the linear fit a red line is used. The data were fitted by the equation $y=A x$ where $\mathrm{A}$ is equal to $12,124 \pm 97$. The $\mathrm{R}^{2}$ correlation factor is 0.99961 . The luminescence emission signals are normalized considering the sensitivity of each single dosimeter. The error propagation theory was applied to uncertainties evaluation starting from statistical counting of luminescence signals.

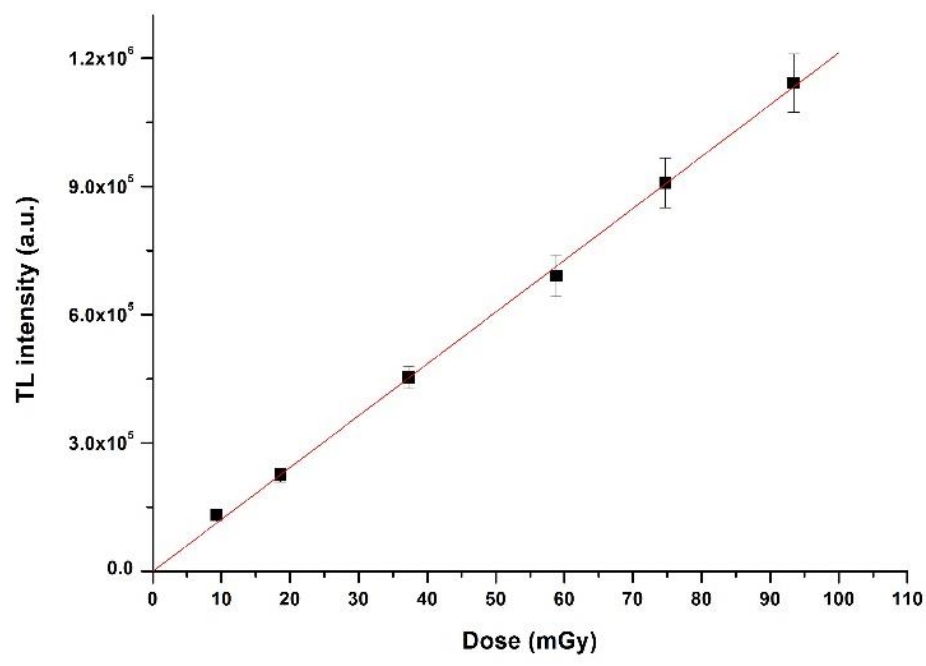

Figure 4. Calibration curve for TLD $100 \mathrm{H}$.

\subsection{Dose Measurements}

Figure $5 \mathrm{a}-\mathrm{c}$ shows the 2D-dose distributions (on the phantom axial plane) obtained by XR-QA2 films. The lowest doses are recorded precisely at the spinal bone that surrounds the spinal cord with his bony parts, while an increasing dose is recorded in the area of the spinal cord.

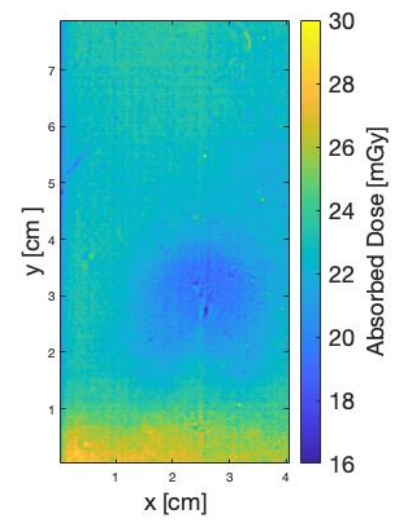

(a)

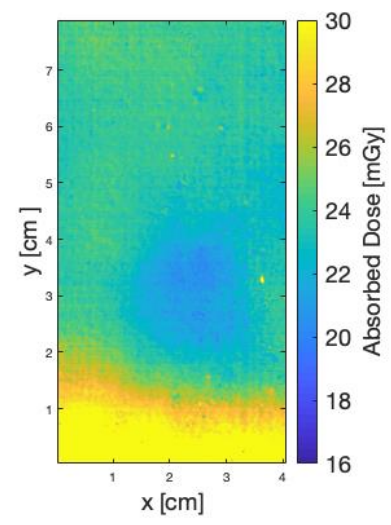

(b)

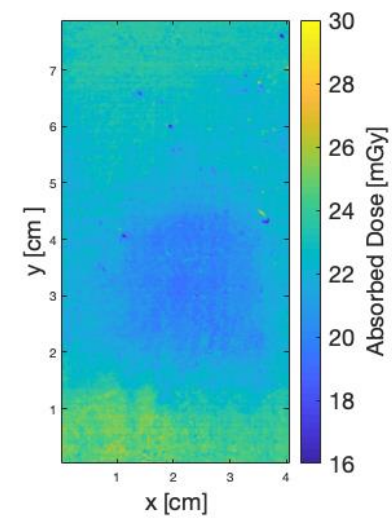

(c)

Figure 5. Film dose responses (mGy) in color wash: (a) 2D-dose distribution in the spinal cord region for the 17th slab; (b) 2D-dose distribution in the spinal cord region for the 18th slab; (c) 2D-dose distribution in the spinal cord region for the 19th slab.

Table 3 shows the maximum, minimum and average dose values obtained for each slab with XR-QA2 films. 
Table 3. Maximum, minimum and average dose value measured with XR-QA2 films for the 17th, 18th and 19th slab.

\begin{tabular}{lccc}
\hline & \multicolumn{3}{c}{ XR-QA2 } \\
\hline & $\begin{array}{c}\text { Max Dose } \\
\text { (mGy) }\end{array}$ & $\begin{array}{c}\text { Min Dose } \\
\text { (mGy) }\end{array}$ & $\begin{array}{c}\text { Mean Dose } \\
\text { (mGy) }\end{array}$ \\
\hline 17th slab & $25.82 \pm 1.35$ & $16.79 \pm 0.88$ & $21.52 \pm 2.93$ \\
18th slab & $26.02 \pm 1.36$ & $17.41 \pm 0.91$ & $20.95 \pm 2.77$ \\
19th slab & $24.05 \pm 1.26$ & $17.45 \pm 0.91$ & $20.55 \pm 2.11$ \\
\hline
\end{tabular}

Table 4 shows the maximum, minimum and average dose values obtained for each slab with TLD.

Table 4. Maximum, minimum and average dose value measured with TLD 100H for the 17th, 18th and 19th slab.

\begin{tabular}{cccc}
\hline & $\begin{array}{c}\text { MLD 100H } \\
(\mathbf{m G y})\end{array}$ & $\begin{array}{c}\text { Min Dose } \\
(\mathbf{m G y})\end{array}$ & $\begin{array}{c}\text { Mean Dose } \\
(\mathbf{m G y})\end{array}$ \\
\hline 17th slab & $27.01 \pm 0.65$ & $17.01 \pm 0.62$ & $22.50 \pm 3.11$ \\
18th slab & $26.21 \pm 0.68$ & $18.81 \pm 0.61$ & $21.90 \pm 2.65$ \\
19th slab & $25.35 \pm 0.59$ & $18.87 \pm 0.58$ & $21.86 \pm 2.17$ \\
\hline
\end{tabular}

Figure $6 \mathrm{a}-\mathrm{c}$ show the average dose values for each TLD measurements as a function of the position and the corresponding average dose value recorded by XR-QA2 films. The XR-QA2 film dose values were extracted using a $15 \times 15$ pixels ROI, centered on the corresponding position of the TLDs (see Figure 2a-c).

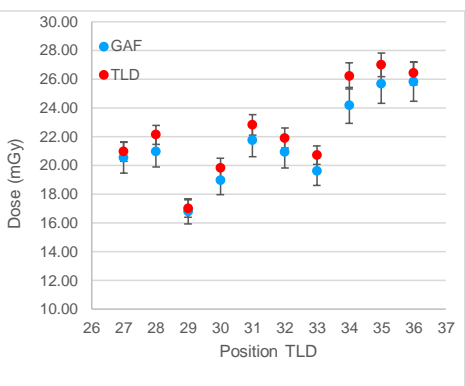

(a)

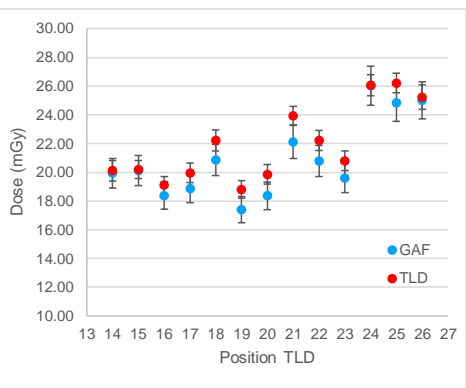

(b)

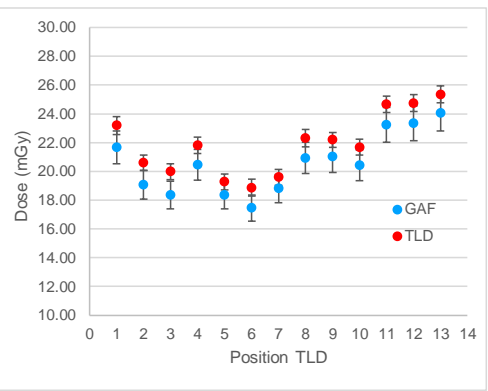

(c)

Figure 6. Average dose values for Gafchromic ${ }^{\mathrm{TM}}$ XR-QA2 (blue dot) and TLD 100H (red dot): (a) dose values trend for the 17th slab; (b) dose values trend for the 18th slab; (c) dose values trend for the 19th slab.

The mean-dose values obtained with the two dosimetric methodologies showed a homothetic behavior, due to the same scan parameters and scattering geometries, with TLD dose values being systematically higher than the Gafchromic ${ }^{\mathrm{TM}}$.

The results are in good agreement with the recent publications by Rampado et al. [9] and Brady et al. [2]. Figure 7a-c shows the slab-by-slab difference in percentage between the two dosimetric methodologies for each numbered TLD position. 


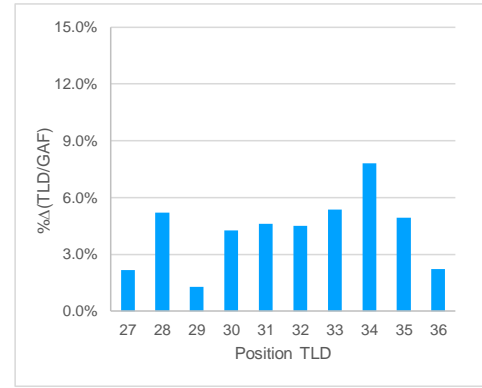

(a)

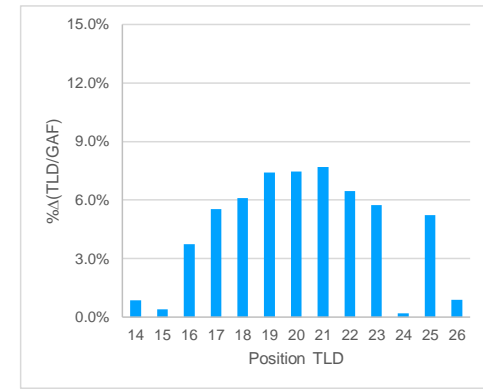

(b)

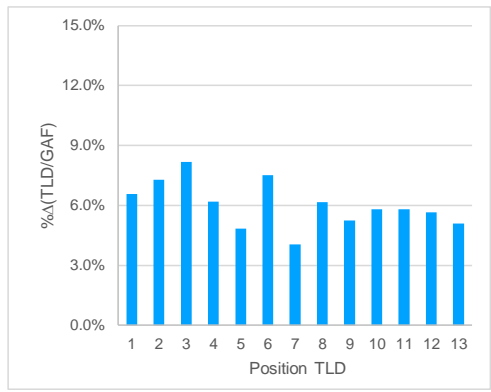

(c)

Figure 7. The difference in percentage for Gafchromic ${ }^{\mathrm{TM}}$ and TLD responses: (a) 17th slab; (b) 18th slab; (c) 19th slab.

Table 5 shows, for each slab, the maximum, minimum and the mean discrepancy in percentage between TLD and Gafchromic ${ }^{\mathrm{TM}}$ dose responses.

Table 5. Maximum, minimum and average percentage discrepancy for the 17th, 18th and 19th slab.

\begin{tabular}{cccc}
\hline & Max $\% \mathbf{\Delta}$ (TLD/GAF) & Min $\% \mathbf{\Delta}$ (TLD/GAF) & Mean $\% \mathbf{\Delta}$ (TLD/GAF) \\
\hline 17th slab & 7.9 & 1.4 & 3.9 \\
18th slab & 7.7 & 0.2 & 4.4 \\
19th slab & 8.2 & 4 & 5.8 \\
\hline
\end{tabular}

The results obtained show higher average percentage discrepancy for the 19th slab compared to the 18th slab and, equally, for the 18th slab compared to the 17th slab.

The CT scan used was equipped with the automatic exposure control (EC) system that reduces the radiation dose to the patient based on their physical dimension and tissue absorption properties [15]. Figure 8a shows the behavior of the current output during the scan length. It emerged that larger current output values correspond to a larger discrepancy between dose values of TLDs and Gafchromic ${ }^{\mathrm{TM}}$ (Figure 8b).

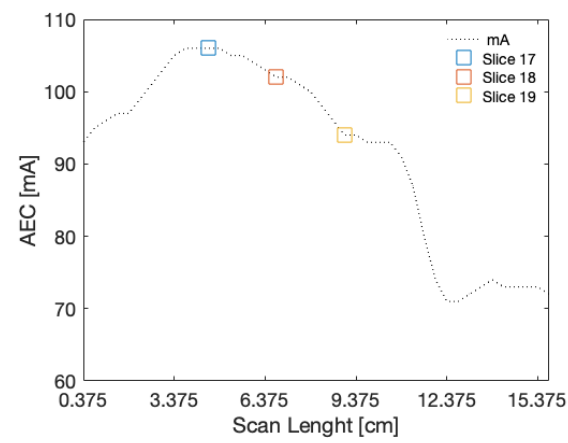

(a)

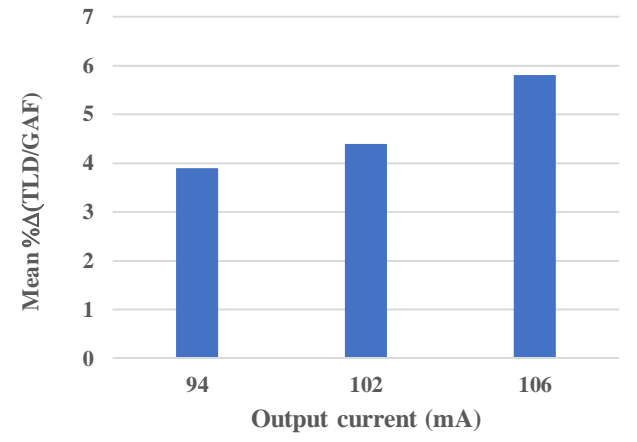

(b)

Figure 8. (a) Current output vs. scan length; the colored squares correspond to the film position between the phantom slabs; (b) mean slice-by-slice $\% \Delta(\mathrm{TLD} / \mathrm{GAF})$ correction factors vs. CT current output.

\section{Conclusions}

In order to consider high dose gradients, e.g., those generated by the bony parts surrounding some organ regions such as the spinal cord, it is helpful to produce high-resolution 2D dosimetric distributions. However, the contributions to the generation of dose distributions are complex and varied, e.g., the angular dependence, the scattering due to the different anatomic structures and the 
EC system that generates variation in the output current during a CT scan. Despite the complexity of these mechanisms, the XR-QA2 provides a good tool to perform patient dosimetry in CT using anthropomorphic phantoms. The dose values obtained using radiochromic films are systematically lower when compared to the dose values obtained with TLDs which are considered among the most accurate dosimeters for punctual dose estimation. The measurements carried out with TLDs show on average a $4-6 \%$ discrepancy with respect to the measurement carried out using Gafchromic ${ }^{\mathrm{TM}}$. It is possible to claim that these values of discrepancy are acceptable since their order of magnitude is comparable to the measured errors. Nevertheless, Figure $8 \mathrm{a}, \mathrm{b}$ shows that the discrepancy between the two methodologies increases for low current output values. The observation of this trend could potentially allow to obtain a slice-by-slice correction factor which depends on the variation of the current output due to the use of the EC system.

Author Contributions: M.P.: conceptualization, investigation, methodology, software, writing original draft; G.S.: conceptualization, data curation, formal analysis, investigation, methodology, writing — review and editing; L.B.T.: project administration, validation; Stefania Mazzaglia: investigation; A.M.G.: conceptualization, validation, supervision, writing-review and editing. All authors have read and agreed to the published version of the manuscript.

Funding: This research received no external funding.

Conflicts of Interest: The authors declare no conflicts of interest.

\section{References}

1. Report CEP08007. The imPACT Group 2009 Buyer's Guided: Multi-Slice CT Scanners. St. George's Healthcare Trust. March 2009. Available online: https://www.scribd.com/document/224289000/Cep-08007 (accessed on 3 July 2020).

2. Brady, S.; Yoshizumi, T.; Toncheva, G.; Frush, D. Implementation of radiochromic film dosimetry protocol for volumetric dose assessments to various organs during diagnostic CT procedures. Med. Phys. 2010, 17, 4782-4792. [CrossRef] [PubMed]

3. Purwaningsih, S.; Lubis, L.E.; Pawiro, S.A.; Soejoko, D.S. Measurement of computed tomography dose profile with pitch variation using Gafchromic XR-QA2 and thermoluminescence dosimeter (TLD). J. Phys. 2016, 694, 012046. [CrossRef]

4. Tomic, N.; Quintero, C.; Whiting, B.; Aldelaijan, S.; Bekerat, H.; Liang, L.; Devic, S. Characterization of calibration curves and energy dependence GafchromicTM XR-QA2 model based radiochromic film dosimetry system. Med. Phys. 2014, 6, 062105. [CrossRef] [PubMed]

5. Dong, S.L.; Chu, T.C.; Lan, G.Y.; Wu, T.H.; Lin, Y.C.; Lee, J.S. Characterization of high-sensitivity metal oxide semiconductor field effect transistor dosimeters system and $\mathrm{LiF}: \mathrm{Mg}, \mathrm{Cu}, \mathrm{P}$ thermoluminescence dosimeters for use in diagnostic radiology. Appl. Radiat. Isot. 2002, 57, 883-891. [CrossRef]

6. Triolo, A.; Brai, M.; Bartolotta, A.; Marrale, M. Glow curve analysis of TLD-100H irradiated with radiation of different LET: Comparison between two theoretical method. Nucl. Instrum. Method. Phys. Res. Sect. A Accel. Spectrom. Detect. Assoc. Equip. 2006, 560, 413-417. [CrossRef]

7. Carinou, E.; Boziari, A.; Askounis, P.; Mikulis, A.; Kamenopoulou, V. Energy dependence of TLD 100 and MCP-N detectors. Radiat. Meas. 2008, 43, 599-602. [CrossRef]

8. Tomic, N.; Devic, S.; DeBlois, F.; Seuntjens, J. Reference radiochromic film dosimetry in kilovoltage photon beams during CBCT image acquisition. Med. Phys. 2020, 37, 1083-1092. [CrossRef] [PubMed]

9. Rampado, O.; Garelli, E.; Ropolo, R. Computed tomography dose measurements with radiochromic films and a flatbed scanner. Med. Phys. 2010, 37, 189-196. [CrossRef] [PubMed]

10. Asero, G.; Greco, C.; Gueli, A.M.; Raffaele, L.; Spampinato, S. Evaluation of spatial resolution in image acquisition by optical flatbed scanners for radiochromic film dosimetry. J. Instrum. 2016, 11, P03024. [CrossRef]

11. Pace, M.; Tonghi, L.B.; Mazzaglia, S.; Stella, G.; Tuvè, C.; Gueli, A.M. 3-D dose distribution for organ dose measurement in CT thoracic exams using Gafchromic ${ }^{\mathrm{TM}}$ XR-QA2 films. J. Instrum. 2019, 14, P09010. [CrossRef] 
12. Giaddui, T.; Cui, Y.; Galvin, J.; Chen, W.; Yu, Y.; Xiao, Y. Characteristics of Gafchromic XRQA2 films for kV image dose measurement. Med. Phys. 2012, 39, 842-850. [CrossRef] [PubMed]

13. Butson, M.J.; Peter, K.N.; Cheung, T.; Metcalfe, P. Radiochromic film for medical radiation dosimetry. Mater. Sci. Eng. R Rep. 2003, 41, 61-120. [CrossRef]

14. Alnawaf, H.; Butson, M.J.; Cheung, T.; Peter, K.N. Scanning orientation and polarization effects for XRQA radiochromic film. Phys. Med. 2010, 26, 216-219. [CrossRef] [PubMed]

15. Spampinato, S.; Gueli, A.M.; Milone, P.; Raffaele, L.A. Dosimetric changes with computed tomography automatic tube-current modulation techniques. Radiol. Phys. Tech. 2018, 11, 184-191. [CrossRef] [PubMed]

16. Gueli, A.M.; Cavalli, N.; De Vincolis, R.; Raffaele, L.; Troja, S.O. Background fog subtraction methods in Gafchromic ${ }^{\circledR}$ dosimetry. Radiat. Meas. 2015, 72, 44-52. [CrossRef]

17. Mahajna, S.; Yossian, D.; Horowitz, Y.S. The effect of thermal annealing on defect structure and thermoluminescence in LiF: Mg, Cu, P. Radiat. Meas. 1995, 24, 395-400. [CrossRef]

18. Stella, G.; Mazzaglia, S.; Pace, M.; Tonghi, L.B.; Tuvè, C.; Gueli, A.M. QA for calibration procedures of TLDs $100 \mathrm{H}$ in low doses range. J. Instrum. 2019, 14, P06023. [CrossRef]

19. Stella, G.; Fontana, D.; Gueli, A.; Troja, S. Historical mortars dating from OSL signals of fine grain fraction enriched in quartz. Geochronometria 2013, 40, 153-164. [CrossRef]

20. Reshes, G.; Druzhyna, S.; Biderman, S.; Eliyahu, I.; Oster, L.; Horowitz, Y.S. Study of the effect of optical bleaching at selected photon energies on the optical absorption and thermoluminescence of LiF: $\mathrm{Mg}, \mathrm{Ti}$ (TLD-100). Radiat. Meas. 2017, 106, 26-29. [CrossRef]

(C) 2020 by the authors. Licensee MDPI, Basel, Switzerland. This article is an open access article distributed under the terms and conditions of the Creative Commons Attribution (CC BY) license (http://creativecommons.org/licenses/by/4.0/). 